\title{
Satisfação com os Cuidados Anestésicos num Hospital Central
}

\author{
Patient Satisfaction with Anesthesia Care in a General Hospital
}

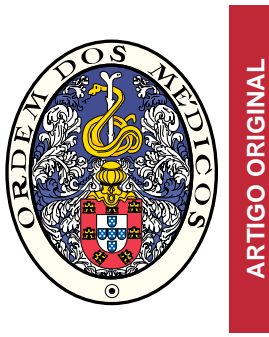

\author{
Ana Catarina MOURA ${ }^{1}$, Maria Amélia FERREIRA ${ }^{1}$, Joselina BARBOSA ${ }^{1}$, Joana MOURÃO ${ }^{2}$ \\ Acta Med Port 2014 Jan-Feb;27(1):33-41
}

RESUMO

Introdução: O nível de satisfação com os cuidados de saúde reflete a qualidade dos cuidados prestados segundo a perspetiva do doente. O objetivo do estudo é avaliar a satisfação dos doentes com os cuidados anestésicos num hospital central português através da aplicação do questionário "Heidelberg Peri-anaesthetic Questionnaire".

Material e Métodos: O questionário foi traduzido e testado segundo critérios de qualidade psicométrica numa amostra de 107 doentes submetidos a cirurgia eletiva com internamento no Hospital de São João. A satisfação total e para cada dimensão foram calculadas. Analisámos as diferenças entre doentes com diferentes níveis de satisfação, identificando potenciais fatores de confundimento.

Resultados: A versão portuguesa do questionário é constituída por 32 itens distribuídos por três dimensões: 'equipa', 'desconforto' e 'medo'. Os valores médios de satisfação para cada dimensão foram $83,4 \% ; 66,8 \%$ e $65,9 \%$, respetivamente. A consistência interna foi demonstrada através de valores alfa de Cronbach de 0,776 a 0,875 nas três dimensões. Os doentes de satisfeitos e insatisfeitos diferiram nas três dimensões, com uma diferença menor em 'equipa'. Na análise multivariada encontrámos influência significativa do género na dimensão 'desconforto'.

Discussão: O questionário apresentou boas características psicométricas. A dimensão 'equipa' inclui três domínios do questionário original.

Conclusões: A sua aplicação revelou elevados níveis de satisfação com a equipa profissional. A insatisfação é sobretudo um reflexo das dimensões 'medo' e 'desconforto', sendo este significativamente menor nos homens.

Palavras-chave: Anestesia; Satisfação do Doente; Qualidade dos Cuidados de Saúde; Questionários; Psicometria; Portugal.

\section{ABSTRACT}

Introduction: The satisfaction level with health care reflects the quality of care from the patient's perspective. The aim of this study is to assess patient satisfaction with anesthesia care in a Portuguese general hospital by using the "The Heidelberg Peri-anaesthetic Questionnaire".

Material and Methods: The questionnaire was translated and tested based on psychometric quality criteria in a sample of 107 patients who underwent elective surgery as inpatients at Hospital de São João. The global satisfaction and for each dimension of care were calculated. We analyzed the differences between patients with different levels of satisfaction, identifying potential confounding factors. Results: The Portuguese version of the questionnaire has 32 items distributed in three dimensions: 'staff', 'discomfort' and 'fear'. The mean values of satisfaction for each dimension were $83.4 \%, 66.8 \%$ and $65.9 \%$, respectively. The internal consistence was demonstrated by a Cronbach's alpha coefficient ranging from 0.776 to 0.875 in the three dimensions. Satisfied and dissatisfied patients differed in the three dimensions, but to a lesser degree in 'staff'. In the multivariate analysis we found significant influence of gender in the 'discomfort' dimension.

Discussion: The questionnaire has good psychometric characteristics. The domain 'staff' includes three domains of the source questionnaire.

Conclusions: Its application revealed high satisfaction levels regarding the staff. Dissatisfaction was mainly seen in the "fear" and "discomfort" dimensions, the latter being significantly lower in males.

Keywords: Anesthesia; Patient Satisfaction; Quality of Health Care; Questionnaires; Psychometrics; Portugal.

\section{INTRODUÇÃO}

O valor dos cuidados de saúde é definido por Michael Porter ${ }^{1}$ como os resultados alcançados em relação ao custo total da prestação de cuidados. Porter valoriza as perspetivas dos doentes e, na avaliação dos cuidados de saúde, aponta para a inclusão daquilo que estes consideram mais relevante. ${ }^{1}$ É baseado nestes pressupostos que Neuman ${ }^{2}$ apresenta a avaliação da satisfação com os cuidados anestésicos. A satisfação é o resultado dos cuidados prestados segundo a perspetiva do doente. Esta medida proporciona uma base para que se possa melhorar os cuidados na

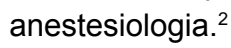

A satisfação é apresentada na literatura como um novo indicador de qualidade dos cuidados de saúde. Correlaciona-se com o comportamento do doente, nomeadamente na adesão ao tratamento e na possibilidade de seguimento. ${ }^{3}$ $\mathrm{Na}$ anestesiologia, a satisfação surge da necessidade de estabelecer novas medidas de avaliação pois "a especialidade tornou-se vítima do seu próprio sucesso". ${ }^{4}$

Segundo a definição clássica, a satisfação é determinada a partir do grau de congruência entre a expectativa do doente e o concretizado. Os autores estão cientes de que se trata de uma medida subjetiva e difícil de avaliar. ${ }^{5}$ Contudo, a sua importância em anestesiologia é reconhecida e vários questionários de satisfação têm sido publicados. ${ }^{6-14}$

1. Faculdade de Medicina. Universidade do Porto. Porto. Portugal.

2. Serviço de Anestesiologia e Terapêutica da Dor. Centro Hospitalar São João. Porto. Portugal.

Recebido: 20 de Agosto de 2013 - Aceite: 22 de Setembro de 2013 | Copyright @ Ordem dos Médicos 2014 
Na sua construção, é fundamental a população estar envolvida de modo a perceber o que é esperado pelo doente e de que depende a sua satisfação. É uma avaliação multidimensional do cuidado, considerando que são abordados aspetos específicos que se acredita contribuírem para a satisfação global. ${ }^{15}$ Para criar questionários válidos e fiáveis, devem ser aplicados testes de qualidade psicométrica. .,16,17 $^{-17}$

Schiff et $\mathrm{al}^{13}$ consideram que mais importante que determinar o nível de satisfação com o serviço prestado, é identificar doentes insatisfeitos. Estes autores construíram e desenvolveram um questionário multidimensional que abrange diferentes fases do cuidado anestésico e procura abordar as possíveis causas de insatisfação dos doentes. O questionário é apresentado como sendo um instrumento válido e fiável, de fácil aplicação no final do período pós-operatório e concebido para ser usado em estudos transversais. $^{13}$

O objetivo do presente estudo é avaliar a satisfação com os cuidados anestésicos num hospital central português. Considerando que em Portugal não foram desenvolvidos questionários multidimensionais de satisfação, será aplicado o questionário acima mencionado.

\section{MATERIAL E MÉTODOS Instrumento}

O instrumento consiste numa adaptação do questionário elaborado por Schiff et al. ${ }^{13} \mathrm{Na}$ construção e validação do "Heidelberg Peri-anaesthetic Questionnaire" estiveram envolvidos vários profissionais de saúde e um largo espectro de doentes de três hospitais na Alemanha. A sua tradução e aplicação foram consentidas.

O questionário é constituído por 38 itens com os quais o doente estabelece um nível de concordância segundo uma escala de Likert de quatro pontos, impossibilitando a escolha de uma resposta central. O sentido da satisfação na escala difere consoante a formulação de cada item.

Os itens estão ordenados cronologicamente de acordo com as fases do internamento e distribuem-se por cinco dimensões: confiança e atmosfera; tratamento dado pela equipa; informação e espera; medo; desconforto. A consistência interna do questionário foi demonstrada através de um valor alfa de Cronbach de 0,79. Os cinco domínios apresentam um valor alfa de Cronbach de 0,42 a 0,79.13

\section{Tradução e Adaptação Transcultural}

A metodologia usada na tradução, de inglês para português, baseou-se na apresentada por Wild et al. ${ }^{18}$ Dois tradutores, incluindo um médico anestesiologista, produziram independentemente duas versões em português, mantendo o nível de leitura e interpretação compatível com o sexto ano de escolaridade proposto no original. De seguida, discutiram os resultados para elaborar uma versão de consenso. Partindo desta versão, foi efetuada uma retroversão por tradutor bilingue que desconhecia o questionário original e os objetivos do estudo.

No final, o questionário passou por uma fase de pré-teste. Uma amostra da população alvo, não incluída nos passos seguintes do estudo, foi abordada após o preenchimento do questionário. Avaliou-se a compreensão dos itens e o grau de dificuldade na escolha da resposta.

\section{Participantes}

A versão portuguesa do questionário foi distribuída no período de 10 de Dezembro de 2012 a 4 de Janeiro de 2013 numa amostra selecionada por conveniência no Serviço de Cirurgia Geral do Hospital de São João no Porto. Os critérios de inclusão foram a idade superior a 18 anos, cirurgia eletiva há pelo menos 12 horas com internamento e ausência de doença neuropsiquiátrica. Os doentes com limitação na leitura ou escrita não foram excluídos do estudo.

Após obtenção do consentimento informado, os questionários foram recolhidos e, para garantir a confidencialidade, procedemos à sua codificação. Recolheram-se as seguintes variáveis: idade, género, estado civil, literacia, risco cirúrgico, ${ }^{19}$ estado físico ASA (American Society of Anesthesiologists), ${ }^{20}$ cirurgia prévia, tipo e duração da anestesia.

O protocolo do estudo foi aprovado pela Comissão de Ética do Hospital São João.

\section{Análise Estatística}

\section{Validade de Constructo e Consistência Interna}

Determinámos a estrutura relacional dos itens através de uma Análise Fatorial Exploratória, utilizando o método de análise de componentes principais seguida de rotação varimax. Optámos por atribuir a média aos itens não respondidos, reforçando os dados da análise. Cargas fatoriais absolutas iguais ou superiores a 0,35 foram consideradas satisfatórias. O número de fatores a reter foi determinado através do critério Scree Plot.

Calculámos as comunalidades no sentido de quantificar a variância de cada item explicada por cada fator extraído. Elevados valores de comunalidades indicam que o método dos componentes principais extraiu uma grande quantidade de variância de um item particular. Excluímos itens que apresentavam comunalidades inferiores a 0,2.

Considerando que o sentido da satisfação na escala de Likert depende da formulação do item, invertemos a pontuação de alguns itens.

Avaliámos a consistência interna do questionário através do cálculo do valor alfa de Cronbach por dimensão e para o total dos itens.

O nível de satisfação para cada dimensão foi obtido através da soma das respostas dos seus itens e convertido em percentagem (satisfação de 0 a 100).

\section{Variáveis de Confundimento}

Realizámos uma análise univariada entre os valores de satisfação de cada dimensão e as características sociodemográficas e clínicas. A associação linear foi determinada através do coeficiente de correlação de Pearson. Categorizámos as variáveis recolhidas nos casos em que se verificavam associações fracas. Conforme adequado, usou-se o Teste t-Student e a Análise de Variância para avaliar a 
significância da diferença entre os valores médios da satisfação e as variáveis qualitativas.

Incluímos num modelo de regressão linear múltipla as variáveis que demonstravam significância na análise univariada (definida como $p<0,2$ ). A associação entre as características dos participantes e as dimensões foi determinada pelos coeficientes de regressão.

\section{Satisfação e Insatisfação}

Estabelecemos o nível de satisfação total através da soma dos valores de satisfação de cada dimensão. Considerámos doentes satisfeitos aqueles que se encontravam um desvio padrão acima da média da satisfação total e doentes insatisfeitos abaixo dessa média. Os restantes não foram incluídos em nenhum dos grupos. ${ }^{13}$

A análise estatística foi realizada no SPSS ${ }^{\circledR}$ (Statistical Package for the Social Sciences) versão 18. O nível de significância foi definido como $p<0,05$. Os valores apresentados correspondem à média ( \pm desvio padrão).

\section{RESULTADOS}

\section{Versão Portuguesa do Questionário}

Considerando que a satisfação envolve conceitos subjetivos, a tradução conceptual terá um papel mais importante que a tradução literal. ${ }^{21}$ Os tradutores produziram duas versões mantendo esta equivalência e estabeleceram uma versão final sem dificuldades. As palavras stress e staff, sem correspondência literal, traduziram-se para 'medo' e 'membros da equipa', respetivamente.

Adaptámos os itens 1 a 6 e 12 para adequar o questionário à situação. Nos primeiros seis itens, referentes ao contacto com o anestesiologista na fase pré-anestésica, poderia existir alguma dificuldade em diferenciar o médico da consulta pré-anestésica com aquele contactado no momento antes da cirurgia. Já que nem todos os doentes têm consulta, optámos por avaliar o segundo contacto, sendo os itens reformulados nesse sentido. Apresentámos o item 12 'The waiting time the morning before the surgery was long' - como 'O tempo de espera no dia da cirurgia foi longo', visto que o período de espera entre a admissão e a cirurgia não é necessariamente a manhã. O questionário original foi comparado com a retroversão e, com exceção das adaptações referidas, não encontramos discrepâncias conceptuais.

Conforme sugerido pelos autores do questionário original, acrescentámos um item referente à qualidade de sono na noite após a cirurgia. ${ }^{13}$ Formulámos a questão da mesma forma que o item que avalia o sono antes da cirurgia e, seguindo a ordem cronológica, colocámos como item 37. Assim, a versão portuguesa é constituída por 39 itens avaliados segundo uma escala de Likert de quatro pontos ( 1 = discordo totalmente; 2 = discordo; 3 = concordo; 4 = concordo totalmente).

O questionário português foi concluído após teste em 15 doentes. Os doentes referiram que a escolha da resposta exigia alguma atenção inicial pelas frases não estarem formuladas no mesmo sentido da satisfação. Contudo, notam que no final se revelava um questionário fácil de com- pletar, demorando em média dez minutos. Foram capazes de responder a todos os itens com a exceção de dois doentes, que não responderam aos primeiros seis itens por não identificarem o anestesiologista.

A versão portuguesa do questionário é apresentada na Tabela 1.

\section{Participantes}

Foram abordados 119 doentes, dos quais 107 (90\%) entregaram o questionário preenchido juntamente com o consentimento informado. As razões mencionadas pelos doze doentes que se recusaram a preencher foram cansaço, dor e falta de interesse.

As idades dos participantes variam entre 18 e 82 anos (mediana 50, média 51,71 ( \pm 14$)$ ), 32\% encontra-se acima dos 60 anos e a maioria são mulheres (59\%). Relativamente à escolaridade, $43 \%$ tem um nível inferior ao nono ano, $34 \%$ terminou o quarto ano e um doente diz ser analfabeto. Apenas $9 \%$ dos participantes foram submetidos a anestesia loco-regional, sendo que a maioria $(81 \%)$ foi submetida a anestesia geral balanceada. A anestesia geral inalatória e geral endovenosa contaram com cinco doentes cada e a anestesia combinada com um doente.

As restantes características sociodemográficas e clínicas são apresentadas na Tabela 2 .

Os doentes completaram o questionário 46 ( \pm 55 ) horas após o final da cirurgia, permanecendo no recobro 198 $( \pm 360)$ minutos.

\section{Análise de Itens}

O sentido da satisfação da escala Likert difere consoante o item. Nos itens 2, 6, 9, 18 a 21, 23 a 25 e 34 a 39, o número quatro (concordo totalmente) corresponde ao máximo de satisfação alcançado e o número um (discordo totalmente) ao mínimo de satisfação. Os restantes itens são classificados no sentido inverso. Na Tabela 1 é apresentada a média e desvio padrão das respostas obtidas.

Dezanove dos itens do questionário foram sempre completados pelos 107 participantes. Os restantes têm menos de $3 \%$ de respostas em falta, com exceção dos itens 1 a 6 , $18,21,24$ e 25 . Relativamente aos primeiros seis itens, os $8 \%$ dos doentes que optaram não responder, assim como os $6 \%$ que não responderam ao item 18 , tendem a coincidir. Estes itens referem-se ao contacto com o anestesiologista antes da cirurgia. Quanto ao item 21, referente à recuperação da consciência após a anestesia, os doentes que não responderam (5\%) não foram submetidos a anestesia geral. Por fim, aqueles que não respondem ao item 14 (4\%) e item 25 (14\%), relativo ao alívio da dor, revelam, através das perguntas anteriores, que a dor no pós-operatório foi pouca ou nula.

\section{Validade de Constructo e Consistência Interna}

$\mathrm{Da}$ análise, resultaram três fatores que explicavam $42,6 \%$ da variância total. O teste de esfericidade de Bartlett demonstrou resultados estatisticamente significativos $(p<$ $0,001)$, indicando que os itens partilhavam uma variância 
Tabela 1 - Versão Portuguesa do Questionário: análise descritiva, dimensionalidade e consistência interna

\begin{tabular}{l} 
Itens - conteúdo \\
\hline 1. Antes da cirurgia, o tempo de espera pelo anestesiologista foi longo. \\
2. Antes da cirurgia, o contacto com o anestesiologista foi efetuado num ambiente \\
agradável.
\end{tabular}

3. O anestesiologista, que o contactou antes da cirurgia, deveria ser mais simpático.

4. O anestesiologista, que o contactou antes da cirurgia, parecia estar com pressa.

5. O anestesiologista, que o contactou antes da cirurgia, não deu informação suficiente.

6. A informação dada pelo anestesiologista, que o contactou antes da cirurgia, foi fácil de perceber.

7. O medo da anestesia foi importante para si.

8. O medo da cirurgia foi importante para si.

9. Na noite antes da cirurgia sentiu-se calmo.

10. A cirurgia foi adiada para outro dia.

11. Antes da cirurgia sentiu um medo incontrolável.

12. O tempo de espera no dia da cirurgia foi longo.

13. Sentir-se sozinho/a incomodou-o/a.

14. O medo ou agitação no momento antes da anestesia foi importante

15. A sede antes da anestesia foi um problema para si.

16. Sentiu frio ou tremor na sala onde foi anestesiado/a.

17. Dor antes da anestesia causou-lhe ansiedade.

18. A anestesia decorreu exatamente como o anestesiologista lhe tinha explicado.

19. O ambiente na sala onde foi anestesiado/a era agradável.

20. Os membros da equipa cuidaram bem de si e foram prestáveis enquanto era anestesiado/a.

21. O acordar da anestesia foi confortável.

22. Depois de acordar da anestesia, sentiu dor na zona onde foi operado/a.

23. Não teve dor nenhuma ou quase nenhuma noutras áreas do corpo após a cirurgia (por exemplo, cabeça).

24. Os membros da equipa mostraram que estavam verdadeiramente preocupados com a sua dor.

25. Os membros da equipa rapidamente aliviaram a sua dor.

26. As náuseas ou vómitos foram um problema após a anestesia.

27. A rouquidão ou dor de garganta foi um problema após a anestesia.

28. A fraqueza muscular foi um problema após a anestesia.

29. A sede foi um problema após a anestesia.

30. Uma necessidade urgente de urinar foi um problema para si.

31. A sensação de frio ou tremor foi um problema após a anestesia.

32. Foi difícil respirar após a anestesia.

33. O cansaço ou a incapacidade de concentração foi um problema após a anestesia.

34. Imediatamente após acordar da anestesia, os membros da equipa estavam disponíveis para o/a ajudarem.

35. Os membros do recobro ou unidade de cuidados intensivos eram simpáticos.

36. A recuperação após a anestesia correu bem.

37. Na noite após a cirurgia sentiu-se calmo.

38. Sentiu que podia confiar na equipa de anestesia.

39. Pôde ter a certeza que o anestesiologista tomava as decisões tendo em conta o melhor interesse do doente.

Média (DP)
$1,62(0,87)$
$3,57(0,54)$

$1,42(0,70)$

$1,23(0,47)$

$1,67(0,82)$

$3,62(0,53)$

$2,07(1,03)$

$2,57(1,00)$

$2,56(1,10)$

$1,37(0,91)$

$1,78(0,95)$

$2,03(1,04)$

$1,52(0,72)$

$2,11(0,99)$

$2,01(1,05)$

$1,84(1,01)$

$1,52(0,81)$

$3,29(0,81)$

$3,20(0,67)$

$3,62(0,55)$

$3,11(0,80)$

$2,67(1,03)$

$2,97(1,04)$

$3,41(0,73)$

$3,41(0,64)$

$1,93(1,14)$

$2,33(1,10)$

$2,12(1,04)$

$2,56(1,20)$

$1,91(1,10)$

$1,78(0,99)$

$1,65(0,86)$

$2,09(1,01)$

$3,64(0,54)$

$3,69(0,47)$

$3,41(0,57)$

$3,18(0,87)$

$3,58(0,54)$

$3,59(0,55)$
Dimensões - peso fatorial

Equipa

Desconforto

Medo

$-0,439$

$---$

$--$

$-$

$-0,461$

$-0,537$

0,398

0,371

$-0,549$

0,607

---

-- $\quad$--- $\quad 0,622$

$--$

0,798

$--$

$--$

$-0,515$

$-$

0,753

-

$-$

$-$

$--$

$\cdots$

0,723

0,454

0,651

$--$

$-0,577$

$-0,363$

0,726

0,683

--.

---

$--$

0,655

0,466

-- $\quad 0,580$

-.- $\quad 0,532$

---

-

$---$

0,383

$--$

0,412

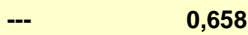

0,416

0,802

0,354

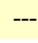

-$$
\text { - }
$$$$
--
$$$$
--
$$

\begin{tabular}{|c|c|c|c|}
\hline Variância & $25,7 \%$ & $10,3 \%$ & $6,6 \%$ \\
\hline a de Cronbach & 0,875 & 0,776 & 0,796 \\
\hline
\end{tabular}




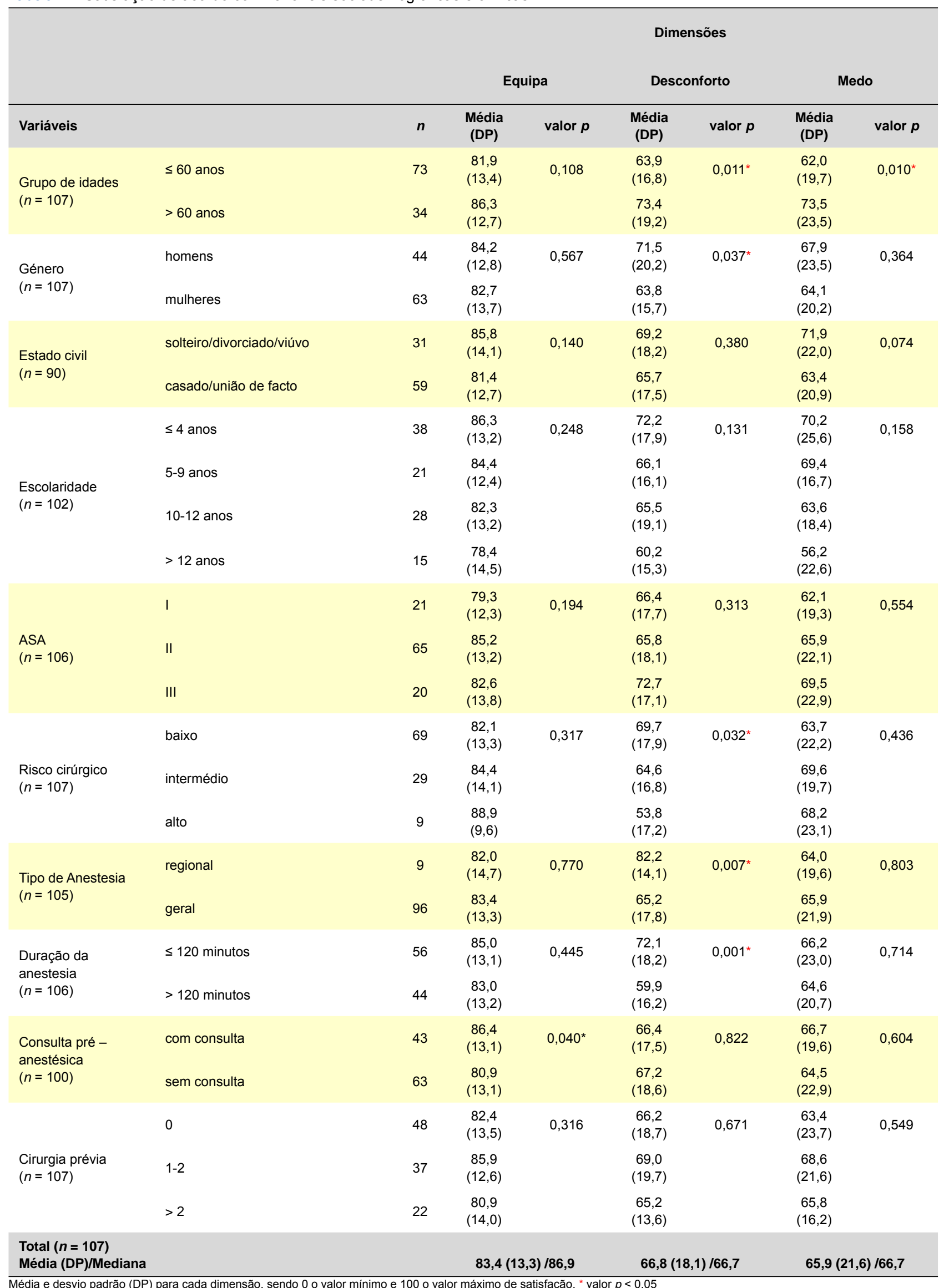


em comum. O teste de Kaiser-Meyer-Olkin, medida de adequação da amostra, obteve um valor de 0,755 , sugerindo que as variáveis medem mais que uma componente. Os itens $2,10,12,16,17,22$ e 30 foram eliminados da análise por apresentarem comunalidades baixas, restando 32 itens (Tabela 1).

De acordo com a carga fatorial (Tabela 1), o primeiro componente é constituído por 14 itens (1, 3-6, 18-20, 24, $25,34,35,38$ e 39 ) e explica $25,7 \%$ da variância. Já o segundo componente, com 11 itens (15, 21, 23, 26-29, 32, $33,36,37)$, explica $10,3 \%$ da variância. Os restantes sete itens $(7-9,11,13,14,31)$ fazem parte do terceiro componente que explica $6,6 \%$ da variância. Os componentes têm valores próprios de 8,2; 3,3 e 2,1 respectivamente. Os itens apresentam uma baixa carga fatorial (inferior a 0,35) para as dimensões a que não pertencem, com exceção dos itens 3, 4 e 36.

Conforme a distribuição apresentada, a primeira dimensão foi denominada 'equipa', a segunda 'desconforto' e a terceira 'medo'.

$\mathrm{Na}$ avaliação da consistência interna, o questionário apresentou um valor alfa de Cronbach de 0,7 para a totalidade dos itens. A dimensão 'equipa' obteve 0,875; a dimensão 'medo' 0,776 e a dimensão ‘desconforto' 0,796.

\section{Variáveis de Confundimento}

Os valores médios de satisfação foram $83,4 \%$ para 'equipa', 66,8\% para 'desconforto' e 65,9\% para 'medo' (Tabela 2$)$. O valor máximo (100\%) representa o máximo de satisfação com a equipa, nenhum desconforto e nenhum medo.

A análise univariada demonstrou que a dimensão 'equipa' é influenciada pela consulta pré-anestésica $(p=0,040)$.
Doentes com consulta têm valores mais elevados de satisfação com a equipa. $O$ 'desconforto' é influenciado pelo género $(p=0,037)$, idade $(p=0,011)$, risco cirúrgico ( $p=$ $0,032)$, tipo de anestesia $(p=0,007)$ e duração da anestesia $(p=0,001)$. Os homens, idade superior a 60 anos, baixo risco cirúrgico, anestesia regional e duração da anestesia inferior a 120 minutos conduzem a menos desconforto. A dimensão 'medo' sofre também influência da idade ( $p=$ $0,010)$. Doentes com mais de 60 anos apresentam valores de satisfação mais elevados, indicando menos medo. Os valores médios de satisfação de acordo com as características dos doentes são apresentados na Tabela 2.

Após análise multivariada, apenas o género manteve o efeito significativo ( $p=0,047)$ em 'desconforto'. Os homens sentiram menos desconforto que as mulheres (coeficiente de regressão = 8,7; intervalo de confiança 95\%:[0,1; 17,3]). Nas dimensões 'equipa' e 'medo', quando ajustado para outras variáveis, não foram encontrados efeitos significativos da consulta pré-anestésica nem da idade, respectivamente.

\section{Satisfação e Insatisfação}

Relativamente à satisfação total, os doentes classificados como insatisfeitos com os cuidados anestésicos apresentam uma mediana (intervalo interquartil [limites]) de $61 \%(55-67 \%[40,70])$ e os doentes satisfeitos uma mediana de $91 \%$ (88-95 [85,99]). Os dois grupos diferem nas três dimensões, embora com diferença menor em 'equipa' (Fig. 1).

\section{DISCUSSÃo}

O questionário de satisfação com os cuidados perianestésicos disponibilizado na literatura por Schiff et al $^{13}$ foi

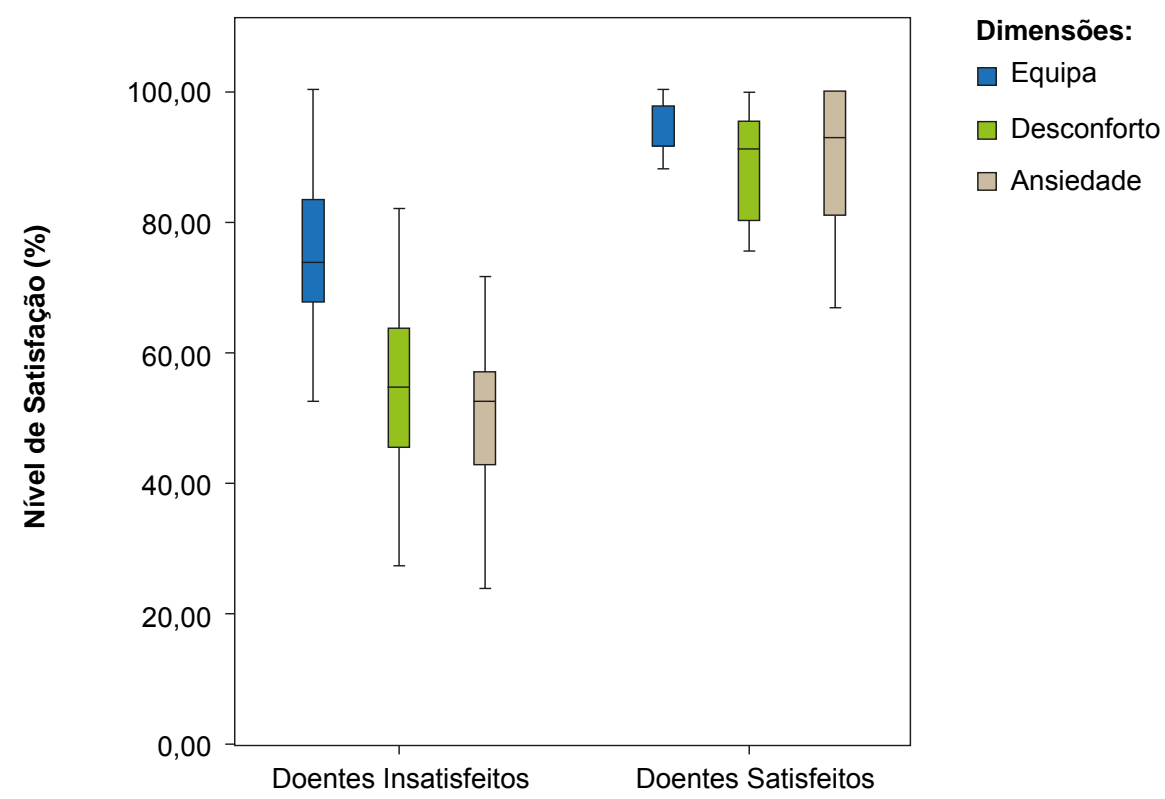

Figura 1 - Dimensões em doentes Satisfeitos e Insatisfeitos. Caixa representa intervalo interquartil, linha horizontal a mediana, e linha vertical os limites mínimo e máximo. Nível de satisfação para cada dimensão, sendo $100 \%$ o máximo de satisfação. 
adaptado para que pudesse ser aplicado num hospital português. Apesar de o ideal na avaliação da satisfação fosse incluir a população alvo na construção de um questionário, é um processo moroso que impossibilita a comparação entre populações. ${ }^{21}$ Desta forma, selecionámos um questionário europeu com validade demonstrada para garantir que os aspetos abordados fossem os mais relevantes. A tradução partiu da versão inglesa publicada, embora tenha sido construído em alemão. A mera adaptação transcultural não garantiria que as características psicométricas fossem preservadas, mas neste contexto tornou-se fundamental o seu teste numa amostra de 107 doentes submetidos a cirurgia eletiva.

Obtivemos uma boa taxa de resposta (90\%) e grande parte do questionário (59\%) foi sempre preenchido. Ao analisarmos os itens com maior número de respostas em falta, concluímos que os doentes que não responderam ao item $21(5 \%)$, relativo ao conforto na recuperação da consciência, foram submetidos a anestesia regional. De facto, os autores do questionário original notam que estes doentes podem encontrar algumas limitações no preenchimento. ${ }^{13}$ Consideramos que o item não deva ser contabilizado neste grupo de doentes em estudos futuros. Relativamente aos que não avaliaram o contacto com o anestesiologista antes da cirurgia, embora a percentagem seja baixa, poderá evidenciar um viés nas respostas dos restantes doentes na avaliação da equipa médica. Já na fase pré-teste verificámos que parte não foi capaz de identificar o seu médico na equipa. Le May et $\mathrm{al}^{22}$ descrevem que $22 \%$ dos doentes envolvidos na construção do seu questionário não se recordavam do contacto prévio com o médico. Sabemos que o reconhecimento do papel do anestesiologista é um tema que esteve sempre presente ao longo dos anos da existência da especialidade ${ }^{23}$ e que atualmente parece continuar subestimado. ${ }^{24}$ Estes resultados podem de certa forma refletir falhas na comunicação entre médico e doente. Desta forma, propomos a inclusão de uma questão inicial para que seja possível identificar estes doentes e avaliar a sua satisfação separadamente.

$\mathrm{Na}$ análise estatística, os itens da versão portuguesa do questionário foram distribuídos por três dimensões, ao contrário das cinco dimensões determinadas no questionário original. ${ }^{13} \mathrm{~A}$ diferença consta na dimensão 'equipa' que inclui os itens pertencentes a três dimensões do questionário alemão (Schiff, comunicação pessoal): 'informação e espera', 'confiança e atmosfera' e 'tratamento dado pela equipa'. Os resultados obtidos na amostra estudada não permitiram distinguir os diferentes domínios do serviço prestado pela equipa. As respostas a estes itens estão fortemente correlacionadas e evoluem no mesmo sentido da satisfação, dificultando esta distinção.

Os itens incluídos em 'medo' e 'desconforto' foram igualmente agrupados pelos autores do questionário original, com exceção de três itens. Um refere-se à sensação de frio ou tremor (item 31), cujos participantes associaram a 'medo' e não a 'desconforto' que acreditamos ser a verdadeira intenção. Inversamente, os itens referentes à recupe- ração da anestesia (itens 21 e 36), foram incluídos em 'desconforto' e não em 'tratamento dado pela equipa' segundo os autores. De acordo com a versão inglesa disponibilizada, a frase parece apoiar a inclusão do item na primeira dimensão. Por fim, o item criado com a intenção de avaliar a qualidade de sono na noite após a cirurgia (item 37), apesar de formulação igual ao item 9 incluído em 'medo', correlacionou-se com os itens pertencentes a 'desconforto'. Concluímos que a noite após a cirurgia é dominada pelo desconforto físico resultante da cirurgia e a noite anterior dominada pelo medo.

Eliminámos sete itens por apresentarem comunalidades baixas (Tabela 2), dois dos quais avaliam o tempo de espera, contudo, não acreditamos que seja relevante para a satisfação com os cuidados anestésicos. De acordo com a realidade hospitalar onde teve lugar o estudo, caso a cirurgia seja adiada (item 10) ou o tempo de espera seja longo (item 12), o doente é avisado antes do internamento. Para além disso, a espera não é exclusivamente dependente da anestesiologia. Estudos futuros com amostras maiores e reformulação de alguns itens poderão criar um modelo mais estável, capaz de incluir os itens excluídos e acrescentar dimensionalidade ao instrumento.

O questionário apresentou boas características psicométricas que permitem considerar válidos para a amostra estudada os resultados da sua aplicação. No que refere à homogeneidade dos itens, determinámos em cada dimensão valores de Cronbach superiores a 0,7 , considerados suficientes por diversos autores. ${ }^{25}$ Demonstrar a fiabilidade do instrumento através da repetição do questionário é controverso nestes estudos. ${ }^{9}$ Sendo duas semanas o tempo mínimo entre testes, o segundo teste seria afetado por eventos relacionados com a cirurgia, ${ }^{8,13}$ como observado por Lemos et $\mathrm{al}^{26}$ num hospital universitário português.

Comparando os resultados com os apresentados por Schiff et $\mathrm{al}^{13}$ os valores de satisfação total foram ligeiramente inferiores. Sabemos que valores altos de satisfação são consistentemente encontrados na literatura, ${ }^{9}$ incluindo estudos desenvolvidos em Portugal. ${ }^{26-28}$ Estes valores geralmente resultam de um único item de avaliação, sendo posteriormente correlacionados com potenciais fatores preditivos ou comparados entre grupos pré-definidos. No entanto, os doentes raramente indicam estar insatisfeitos, dificultando possíveis conclusões. Por este motivo, a satisfação deve ser avaliada através de instrumentos multidimensionais e a sua interpretação baseada na análise individual das dimensões, sendo estas as responsáveis pela satisfação. ${ }^{4,9,16}$ Porém, continua presente o viés de desejabilidade social, ou seja, a tendência para responder de forma socialmente aceitável ou positiva. ${ }^{4,29}$ Este viés foi minimizado com a entrega do questionário, não pelo médico, mas pelo investigador principal, que garantiu a confidencialidade e não esteve presente durante o preenchimento. ${ }^{9,14}$

Os grupos de doentes 'satisfeitos' e 'insatisfeitos' diferiram nas três dimensões do cuidado, embora a dimensão 'equipa' apresentasse também valores elevados nos doentes insatisfeitos. Tal como demonstrado por Schiff et $\mathrm{al}^{13} \mathrm{a}$ 
insatisfação é sobretudo um reflexo das dimensões 'medo' e 'desconforto'. Contudo, a dimensão "informação e espera" estabelecida pelos autores também contribuiu para a insatisfação.

Sintetizando, não foi possível diferenciar os três componentes do cuidado anestésico incluídos em 'equipa', dimensão que obteve o mais alto nível de satisfação. Considerando a incerteza em relação ao número de doentes que identifica o anestesiologista na equipa, podemos avançar com a hipótese de não serem capazes de avaliar criticamente esta área do cuidado. Os doentes não só tendem a aprovar os cuidados que recebem, ${ }^{30,31}$ como também não conhecem suficientemente o papel do anestesiologista como médico para permitir esta avaliação. ${ }^{24}$ Doentes cientes da responsabilidade do profissional poderão ser mais capazes de avaliar os cuidados prestados. Defendemos que o anestesiologista deva procurar educar individualmente os doentes durante a visita pré-anestésica relativamente ao seu papel no perioperatório e estabelecer uma relação de confiança com o doente. ${ }^{24}$ No entanto, não podemos excluir a hipótese de os doentes identificarem o médico e avaliarem positivamente a equipa profissional.

$\mathrm{Na}$ literatura apenas estão disponíveis alguns questionários multidimensionais de satisfação baseados em critérios de qualidade psicométrica. ${ }^{6-14,31}$ Tanto quanto conseguimos apurar, foi já aplicado em Portugal um destes questionários ${ }^{6}$ na comparação de tipos de anestesia oftalmológica, embora não validado. ${ }^{32}$ Notamos também que foi elaborado e testado num hospital universitário português um questionário de satisfação materna após anestesia, não sendo clara a metodologia de construção. ${ }^{33}$ Por se tratar de instrumentos diferentes, construídos em diferentes contextos de cuidado, os seus resultados são difíceis de comparar. Contudo, a informação, comunicação e relação de confiança entre médico e doente são sempre avaliadas. ${ }^{9,10}$ Tal como apresentado neste estudo, Capuzzo e colaboradores $^{10}$ estabeleceram uma única dimensão para esta avaliação e demonstraram ainda ser o elemento do cuidado mais valorizado pelos doentes. Outros autores $8,9,12,14$ distinguiram informação de relação entre médico e doente, constituindo duas dimensões distintas. Nestes estudos, a dimensão 'informação' obteve sempre dos níveis mais baixos de satisfação, ao contrário de 'relação entre médico e doente'/'atenção'/'confiança' que revelou valores de satisfação mais elevados. A dimensão 'medo' é também frequentemente avaliada e geralmente contribui para a insatisfação dos doentes. ${ }^{12-14}$ Em relação a dor e desconforto, segundo Caljouw et $\mathrm{al}^{12}$ mais importante que a identificar é avaliar a forma como os profissionais lidam com o problema. Estes autores verificaram que a satisfação não se correlacionou com a dor, náuseas ou vómitos, mas foi determinada pela perspetiva dos doentes em relação à competência profissional. ${ }^{12}$

Relativamente ao efeito das características dos participantes na satisfação, parece existir alguma tendência em estudos prévios para que as mulheres e os doentes mais novos apresentem valores de satisfação menores..$^{8-14,26}$ No entanto, apenas parte destes trabalhos apresentam uma análise multivariada dos dados..$^{9,11,13}$ De facto, verificámos maior satisfação numa idade mais avançada nas dimensões 'desconforto' e 'medo'. Após análise multivariada encontrámos apenas influência significativa do género em 'desconforto', contudo, foram os homens que revelaram maior satisfação.

Aplicações futuras do questionário poderiam incluir novos itens de avaliação da comunicação entre médico e doente, assim como da capacidade do médico em lidar com o desconforto e medo. ${ }^{12} \mathrm{Em}$ relação à análise estatística, poderá ser aplicada a análise fatorial confirmatória, apresentada mais recentemente na construção de questionários multidimensionais. ${ }^{14} \mathrm{~A}$ validade do instrumento seria também reforçada através da aplicação simultânea de escalas visuais analógicas e sua correlação com potenciais dimensões do questionário. ${ }^{8,13} \mathrm{O}$ "Inventário de Estado-Traço de Ansiedade" encontra-se validado na população portuguesa e poderá também ser aplicado com este objetivo. ${ }^{13,34}$

Pretendemos alertar a especialidade para esta nova abordagem de avaliação da qualidade dos cuidados anestésicos. O resultado da aplicação de questionários de satisfação, nomeadamente na comparação de entre tipos de anestesia, analgesia, consulta ou visita pré-anestésica e cirurgia com internamento ou de ambulatório, terá um peso nas tomadas de decisão dos profissionais. Para além disso, segundo Vuori, ${ }^{35}$ "mais importante que avaliar a competência do médico ou dos cuidados de saúde, o verdadeiro objetivo da Medicina não será alcançado se o doente não estiver satisfeito. O grande objetivo dos cuidados é o alcance das expectativas dos doentes. ${ }^{35}$

\section{CONCLUSÃO}

A versão portuguesa do questionário é um instrumento multidimensional com 32 itens capaz de avaliar três domínios do cuidado anestésico: equipa, medo e desconforto. Os níveis mais altos de satisfação referem-se à avaliação da equipa profissional. A insatisfação foi determinada pelo medo e desconforto, sendo este último significativamente menor nos homens.

\section{CONFLITOS DE INTERESSE}

Os autores declaram a inexistência de conflitos de interesse na realização do presente trabalho.

\section{FONTES DE FINANCIAMENTO}

Não existiram fontes externas de financiamento para a realização deste artigo.

\section{AGRADECIMENTOS}

Os autores gostariam de agradecer a Ricardo Soares-dos-Reis por contribuição relevante. 


\section{REFERÊNCIAS}

1. Porter ME. What is value in health care? N Engl J Med. 2010;363:247781.

2. Neuman MD. Patient satisfaction and value in anesthesia care. Anesthesiology. 2011;114:1019-20.

3. Klock PA, Roizen MF. More or better--educating the patient about the anesthesiologist's role as perioperative physician. Anesth Analg. 1996;83:671-2

4. Fung $D$, Cohen MM. Measuring patient satisfaction with anesthesia care: a review of current methodology. Anesth Analg. 1998;87:1089-98.

5. Capuzzo M, Alvisi R. Is it possible to measure and improve patient satisfaction with anesthesia? Anesthesiol Clin. 2008;26:613-26.

6. Dexter F, Aker J, Wright WA. Development of a measure of patient satisfaction with monitored anesthesia care: the lowa Satisfaction with Anesthesia Scale. Anesthesiology. 1997;87:865-73.

7. Auquier P, Blache JL, Colavolpe C, Eon B, Auffray JP, Pernoud N, et al. Échelle de vécu périopératoire de l'anesthésie. I - Construction et validation. Ann Fr Anesth Reanim. 1999;18:848-57.

8. Auquier P, Pernoud N, Bruder N, Simeoni MC, Auffray JP, Colavolpe C, et al. Development and validation of a perioperative satisfaction questionnaire. Anesthesiology. 2005;102:1116-23.

9. Heidegger T, Husemann Y, Nuebling M, Morf D, Sieber T, Huth A, et al. Patient satisfaction with anaesthesia care: development of a psychometric questionnaire and benchmarking among six hospitals in Switzerland and Austria. Br J Anaesth. 2002;89:863-72.

10. Capuzzo M, Landi F, Bassani A, Grassi L, Volta CA, Alvisi R. Emotional and interpersonal factors are most important for patient satisfaction with anaesthesia. Acta Anaesthesiol Scand. 2005;49:735-42.

11. Capuzzo M, Gilli G, Paparella L, Gritti G, Gambi D, Bianconi M, et al. Factors predictive of patient satisfaction with anesthesia. Anesth Analg. 2007;105:435-42.

12. Caljouw MA, van Beuzekom M, Boer F. Patient's satisfaction with perioperative care: development, validation, and application of a questionnaire. $\mathrm{Br}$ J Anaesth. 2008;100:637-44.

13. Schiff JH, Fornaschon AS, Frankenhauser S, Schiff M, Snyder-Ramos SA, Martin E, et al. The Heidelberg Peri-anaesthetic Questionnaire -development of a new refined psychometric questionnaire. Anaesthesia. 2008;63:1096-104

14. Mui WC, Chang CM, Cheng KF, Lee TY, Ng KO, Tsao KR, et al. Development and validation of the questionnaire of satisfaction with perioperative anesthetic care for general and regional anesthesia in Taiwanese patients. Anesthesiology. 2011;114:1064-75.

15. Jenkinson C, Coulter A, Bruster S, Richards N, Chandola T. Patients' experiences and satisfaction with health care: results of a questionnaire study of specific aspects of care. Qual Saf Health Care. 2002;11:335-9.

16. Sitzia J. How valid and reliable are patient satisfaction data? An analysis of 195 studies. Int J Qual Health Care. 1999;11:319-28.

17. Le May S, Hardy JF, Taillefer MC, Dupuis G. Patient satisfaction with anesthesia services. Can J Anaesth. 2001;48:153-61.

18. Wild D, Grove A, Martin M, Eremenco S, McElroy S, Verjee-Lorenz A, et al. Principles of Good Practice for the Translation and Cultural Adaptation Process for Patient-Reported Outcomes (PRO) Measures: report of the ISPOR Task Force for Translation and Cultural Adaptation. Value Health. 2005;8:94-104.
19. Poldermans D, Bax JJ, Boersma E, De Hert S, Eeckhout E, Fowkes $\mathrm{G}$, et al. Guidelines for pre-operative cardiac risk assessment and perioperative cardiac management in non-cardiac surgery: the Task Force for Preoperative Cardiac Risk Assessment and Perioperative Cardiac Management in Non-cardiac Surgery of the European Society of Cardiology (ESC) and endorsed by the European Society of Anaesthesiology (ESA). Eur J Anaesthesiol. 2010;27:92-137.

20. Aronson WL, McAuliffe MS, Miller K. Variability in the American Society of Anesthesiologists Physical Status Classification Scale. AANA J. 2003;71:265-74.

21. Beaton DE, Bombardier C, Guillemin F, Ferraz MB. Guidelines for the process of cross-cultural adaptation of self-report measures. Spine. 2000;25:3186-191.

22. Le May S, Hardy JF, Harel F, Taillefer MC, Dupuis G. Patients' perceptions of cardiac anesthesia services: a pilot study. Can J Anaesth. 2001;48:1127-42

23. Klafta JM, Roizen MF. Current understanding of patients' attitudes toward and preparation for anesthesia: a review. Anesth Analg. 1996;83:1314-21.

24. Gottschalk A, Seelen S, Tivey S, Gottschalk A, Rich G. What do patients know about anesthesiologists? Results of a comparative survey in an U.S., Australian, and German university hospital. J Clin Anesth. 2013;25:85-91

25. Terwee CB, Bot SD, de Boer MR, van der Windt DA, Knol DL, Dekker $J$, et al. Quality criteria were proposed for measurement properties of health status questionnaires. J Clin Epidemiol. 2007;60:34-42.

26. Lemos P, Pinto A, Morais G, Pereira J, Loureiro R, Teixeira S, et al. Patient satisfaction following day surgery. J Clin Anesth. 2009;21:200-5.

27. Barros F, Lages N, Lopez M, Pereira S. Ansiedade e grau de satisfação em anestesia pediátrica. Rev Soc Port Anest. 2005;14:14-23.

28. Cheung L, Matos F, Pinto F, Alves C, Pereira M, Carvalhas J. Top-up epidural com ropivacaína a $0,75 \%$ para cesariana urgente após analgesia epidural contínua com ropivacaína a $0,2 \%$. Rev Anest Reg Ter Dor. 2010:46-52.

29. May LA, Smiley B, Schmidt MG. Comparative denaturing gradient gel electrophoresis analysis of fungal communities associated with whole plant corn silage. Can J Microbiol. 2001;47:829-41.

30. Sitzia J, Wood N. Patient satisfaction: a review of issues and concepts. Soc Sci Med. 1997;45:1829-43.

31. Williams B, Coyle J, Healy D. The meaning of patient satisfaction: an explanation of high reported levels. Soc Sci Med. 1998;47:1351-9.

32. Rodrigues PA, Vale PJ, Cruz LM, Carvalho RP, Ribeiro IM, Martins JL. Topical anesthesia versus sub-Tenon block for cataract surgery: surgica conditions and patient satisfaction. Eur J Ophthalmol. 2008;18:356-60.

33. Matos FM, Gomes A, Eufrásio A, Alves C, Carvalhas J. Satisfação materna após anestesia para cesariana: comparação entre bloqueio epidural e bloqueio subaracnóideu. Rev Anest Reg Ter Dor. 2010:55-9.

34. Telles-Correia D, Barbosa A. Ansiedade e depressão em medicina. Modelos teóricos e avaliação. Acta Med Port. 2009;22:89-98.

35. Heidegger $T$, Nuebling $M$, Saal D, Kreienbuhl G. Patient-centred outcomes in clinical research: does it really matter? $\mathrm{Br} \mathrm{J}$ Anaesth. 2008;100:1-3. 


\section{Satisfação com os Cuidados Anestésicos num Hospital Central \\ Acta Med Port 2014:27:33-41}

Publicado pela Acta Médica Portuguesa, a Revista Científica da Ordem dos Médicos

Av. Almirante Gago Coutinho, 151

1749-084 Lisboa, Portugal.

Tel: +351 218428215

E-mail: submissao@actamedicaportuguesa.com

www.actamedicaportuguesa.com

ISSN:0870-399X | e-ISSN: 1646-0758

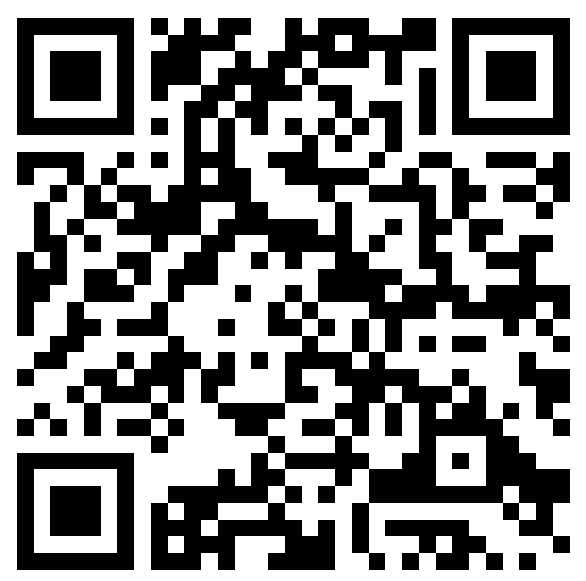

